\title{
Combing NLR, V20 and mean lung dose to predict radiation induced lung injury in patients with lung cancer treated with intensity modulated radiation therapy and chemotherapy
}

\author{
Wen-Yan Pan ${ }^{1,2}$, Chao Bian ${ }^{3}$, Guan-Lian Zou ${ }^{3}$, Cui-Ying Zhang ${ }^{1,2}$, Ping Hai', ${ }^{1,2}$ Ren \\ Zhao $^{1,2}$ and Yan-Yang Wang ${ }^{1,2}$ \\ 'Department of Radiation Oncology, General Hospital of Ningxia Medical University, Yinchuan 750004, Ningxia, China \\ ${ }^{2}$ Cancer Institute, Ningxia Medical University, Yinchuan 750004, Ningxia, China \\ ${ }^{3}$ Graduate School, Ningxia Medical University, Yinchuan 750004, Ningxia, China \\ Correspondence to: Yan-Yang Wang, email: fdwyy1981@hotmail.com, wangyy@nxmu.edu.cn \\ Ren Zhao, email: nxzr1964@hotmail.com
}

Keywords: neutrophil to lymphocyte ratio (NLR), V20, mean lung dose, radiation induced lung injury, lung cancer Received: March 06, 2017

Accepted: June 18, 2017

Published: July 06, 2017

Copyright: Pan et al. This is an open-access article distributed under the terms of the Creative Commons Attribution License 3.0 (CC BY 3.0), which permits unrestricted use, distribution, and reproduction in any medium, provided the original author and source are credited.

\section{ABSTRACT}

The purpose was to evaluate the predictive value of baseline neutrophil to lymphocyte ratio (NLR) level in the incidence of grade 3 or higher radiation induced lung injury (RILI) for lung cancer patients. A retrospectively analysis with 166 lung cancer patients was performed. All of the enrolled patients received chemoradiotherapy at our hospital between April 2014 and May 2016. The Cox proportional hazard model was used to identify the potential risk factors for RILI. In this cohort, the incidence of grade 3 or higher RILI was $\mathbf{2 3 . 8 \%}$. Univariate analysis showed that radiation dose, volume at least received 20Gy (V20), mean lung dose and NLR were significantly associated with the incidence of grade 3 or higher RILI $(P=0.012,0.008,0.012$, and 0.039 , respectively). Multivariate analysis revealed that total dose $\geq 60 \mathrm{~Gy}, \mathrm{~V} 20$ $\geq 20 \%$, mean lung dose $\geq 12 \mathrm{~Gy}$, and NLR $\geq 2.2$ were still independent predictive factors for RILI $(P=0.010,0.043,0.028$, and 0.015 , respectively $)$. A predictive model of RILI based on the identified risk factors was established using receiver operator characteristic curves. The results demonstrated that the combination analysis of V20, mean lung dose and NLR was superior to either of the variables alone. Additionally, we found that the constraint of $\mathrm{V} 20$ and mean lung dose were meaningful for patients with higher baseline NLR level. If the value of V20 and mean lung dose lower than the threshold value, the incidence of grade 3 or higher RILI for the high NLR level patients could be decreased from $63.3 \%$ to $8.7 \%$. Our study showed that radiation dose, V20, mean lung dose and NLR were independent predictors for RILI. Combination analysis of V20, mean lung dose and NLR may provide a more accurate model for RILI prediction.

\section{INTRODUCTION}

As one of dose-limiting factors, radiation induced lung injury (RILI) limits the usage of radiotherapy in lung cancer patients. Although a growing body of evidence has shown that some clinical factors, dosemetric factors and biology factors are associated with the incidence of RILI, there is no consensus about the prediction of RILI in patients with lung cancer until now [1-4]. Therefore, accurately assess individual patient's risk of developing RILI deserves further investigation.

More and more studies suggest that the inflammation background of host had an influence on the incidence of RILI [5-7]. The neutrophil to lymphocyte ratio (NLR), which involves measurement of a subgroup of white blood cells, has been identified as a marker of systemic inflammation. Increased pre-treatment NLR level has been used in combination with other inflammatory markers to 
determine the prognosis of many diseases [8-12]. However, as we known, the relationship between pre-radiotherapy NLR level and RILI has not been well documented before.

In this study, we investigated the prediction role of NLR and other clinical or dosimetric risk factors for grade 3 or higher RILI in 166 patients with lung cancer. All of the enrolled patients received concurrent or sequential chemoradiotherapy in our department. The combination value of NLR and other meaningful factors in the prediction of grade 3 or higher RILI was also determined.

\section{RESULTS}

The features of the final analyzed patients, tumors, and treatments are list in Table 1 . There were 116 men and 35 women, with median age of 60 years (range, 29 to 78 years). Among them, 50.3\% were non-small cell lung cancer (NSCLC), 10.6\% received surgery treatment. Of these 151 patients, 72 received sequential chemoradiotherapy, 79 received concurrent chemoradiotherapy. $57.6 \%$ of these patients received 50 to 60 Gy of radiation, and $42.4 \%$ received equal or more than $60 \mathrm{~Gy}$. The median chemotherapy cycles of the whole study population was 4 (range, 1 to 10 cycles). In total, 36 patients $(23.8 \%)$ developed grade 3 or higher RILI, including two patients died of RILI. These events was frequently observed within one to three months after radiotherapy.

On univariate analysis, total dose, the volume at least received 20Gy (V20), mean lung dose (MLD) and NLR were significantly associated with grade 3 or higher RILI ( $P=0.012,0.008,0.012$, and 0.039 , respectively). These four meaningful factors were then entered into the multivariate analysis. The results of the multivariate analysis revealed that total dose $\geq 60 \mathrm{~Gy}, \mathrm{~V} 20 \geq 20 \%$, $\mathrm{MLD} \geq 12 \mathrm{~Gy}$, and NLR $\geq 2.2$ were still independent predictive factors for grade 3 or higher RILI $(P=0.010$, $0.043,0.028$, and 0.015 , respectively). The results of the univariate and multivariate analysis are shown in Table 2.

In order to establish the predictive model of grade 3 or higher RILI, receiver operating characteristic (ROC) curves were generated for V20, or MLD, or NLR, or the combination of these three factors. The results demonstrated that these three parameters alone were poor in predicting grade 3 or higher RILI. The area under the curve (AUC) values for V20, MLD, and NLR were 0.69 (95\% CI, 0.60-0.78, $P<0.001$ ), 0.72 (95\% CI, 0.62-0.81, $P<0.001)$, and 0.66 (95\% CI, 0.56-0.76, $P=0.004)$, respectively. However, combining all three parameters into a single model improved the predictive ability compared to either of the variables alone, producing an AUC of 0.82 (95\% CI, 0.74-0.89, $P<0.001$ ) (Figure 1).

The benefit of combining analysis of these three meaningful parameters was explored further by using threshold values, which determined by ROC curves. The results showed that the threshold values of V20, MLD, and NLR was $20 \%, 12 \mathrm{~Gy}$, and 2.2, respectively. Using threshold values for V20 and MLD, patients with high NLR level were stratified into 4 subgroups: high V20 and high MLD (group 1), low V20 and high MLD, (group 2), high V20 and low MLD (group 3), and low V20 and low MLD (group 4). The incidence of grade 3 or higher RILI was $63.3 \%, 40.0 \%, 25.0 \%$, and $8.7 \%$ in group 1 to 4 , respectively. The results revealed that the threshold values for V20 and MLD were meaningful for the reduction of probability of grade 3 or higher RILI, especially for the high pre-treatment NLR level lung cancer patients.

\section{DISCUSSION}

In current study, an incidence of $23.8 \%$ was observed for the grade 3 or higher RILI, which is similar to the previous study [13]. And the risk factors for the development of RILI were also assessed in this study. No clinical factors, except for NLR, was found significantly associated with RILI. In regard to the dosimetric factors, radiation dose, V20, and MLD were found to be significantly associated with grade 3 or higher RILI in present study. To improve the predictive ability of RILI, the combined analysis of V20, MLD, and NLR was performed using ROC model. For the determination of total prescription dose was usually depend on the histology or the combined therapy method, it did not entered into the ROC analysis. The results of ROC model demonstrated that combining these three factors improved the predictive ability compared to that with either of the factors alone. Subsequently, we found that the constraint of V20 and MLD were meaningful for the higher baseline NLR level patients. If the value of V20 and MLD lower than the threshold value, the incidence of grade 3 or higher RILI for the high NLR level patients can be decreased from $63.3 \%$ to $8.7 \%$.

The role of dose-volume parameters in predicting RILI were suggested in several previous studies [14-17]. Some dosimetric parameters, including V20 and MLD, were reported had a relationship with the occurrence of RILI. However, the consensus thresholds of these parameters did not draw definitive conclusions [17]. In order to reduce the risk of RILI, V20 should be limit less than 30-35\%, and MLD should be limit less than 20-23 Gy, which recommended by Quantitative Analysis of Normal Tissue Effects in the Clinic (QUANTEC) [17]. In current study, we found that the threshold values of V20 and MLD was 20\% and 12Gy, which is lower than the recommendation of QUANTEC. The reasons of this phenomenon not only include heterogeneity of different study population, but also depend on the radiation therapy techniques and the combined treatment approaches. In current study, all of the patients received intensity modulated radiation therapy (IMRT) and more neoadjuvant chemotherapy patients were enrolled.

Since the dose-volume parameters were population based predictors for RILI, more individualized predictors 
Table 1: Clinicopathologic and dosimetric factors of enrolled lung cancer patients according to different NLR level

\begin{tabular}{|c|c|c|c|}
\hline \multirow{2}{*}{ Characteristics } & \multicolumn{2}{|c|}{ NLR, $(n)$} & \multirow{2}{*}{$P$ value } \\
\hline & $\geq 2.2$ & $<2.2$ & \\
\hline \multicolumn{4}{|l|}{ Age, years } \\
\hline$\geq 60$ & 36 & 43 & \\
\hline$<60$ & 40 & 32 & 0.255 \\
\hline \multicolumn{4}{|l|}{ Sex } \\
\hline Male & 60 & 56 & \\
\hline Female & 16 & 19 & 0.567 \\
\hline \multicolumn{4}{|l|}{ KPS } \\
\hline 90 & 33 & 51 & \\
\hline 70,80 & 43 & 24 & 0.003 \\
\hline \multicolumn{4}{|l|}{ Smoke } \\
\hline Ever & 51 & 42 & \\
\hline Never & 25 & 33 & 0.183 \\
\hline \multicolumn{4}{|l|}{ COPD } \\
\hline Yes & 10 & 8 & \\
\hline No & 66 & 67 & 0.803 \\
\hline \multicolumn{4}{|l|}{ Histology } \\
\hline NSCLC & 40 & 36 & \\
\hline SCLC & 36 & 39 & 0.627 \\
\hline \multicolumn{4}{|l|}{ TNM stage } \\
\hline I-II & 40 & 35 & \\
\hline III & 36 & 40 & 0.517 \\
\hline \multicolumn{4}{|l|}{ PORT } \\
\hline Yes & 11 & 15 & \\
\hline No & 65 & 60 & 0.396 \\
\hline \multicolumn{4}{|l|}{ Dose } \\
\hline$\geq 60 \mathrm{~Gy}$ & 32 & 32 & \\
\hline $50-60$ Gy & 44 & 43 & 1.000 \\
\hline \multicolumn{4}{|l|}{ V20 } \\
\hline$\geq 20 \%$ & 42 & 53 & \\
\hline$<20 \%$ & 34 & 22 & 0.064 \\
\hline \multicolumn{4}{|l|}{ MLD } \\
\hline$\geq 12 \mathrm{~Gy}$ & 40 & 34 & \\
\hline$<12 \mathrm{~Gy}$ & 36 & 41 & 0.417 \\
\hline \multicolumn{4}{|l|}{ Neoadjuvant CT } \\
\hline Yes & 61 & 54 & \\
\hline No & 15 & 21 & 0.257 \\
\hline \multicolumn{4}{|l|}{ Concurrent CT } \\
\hline Yes & 38 & 41 & \\
\hline No & 38 & 34 & 0.626 \\
\hline \multicolumn{4}{|l|}{ Adjuvant CT } \\
\hline Yes & 35 & 25 & \\
\hline No & 41 & 50 & 0.135 \\
\hline
\end{tabular}

Abbreviations: NLR, neutrophil to lymphocyte ratio; KPS, Karnofsky scores; COPD, chronic obstructive pulmonary disease; NSCLC, non-small cell lung cancer; SCLC, small cell lung cancer; PORT, post-operative radiotherapy; V20, volume at least received 20Gy; MLD, mean lung dose; CT, chemotherapy. 
Table 2: Univariate and multivariate analysis of clinicopathologic and dosimetric factors associated with the incidence of grade 3 or higher RILI

\begin{tabular}{|c|c|c|c|c|c|c|c|}
\hline \multirow{2}{*}{\multicolumn{2}{|c|}{$\begin{array}{l}\text { Clinicopathologic and dosimetric } \\
\text { factors }\end{array}$}} & \multicolumn{3}{|c|}{ Univariable analysis } & \multicolumn{3}{|c|}{ Multivariable analysis } \\
\hline & & \multirow{2}{*}{$\begin{array}{c}\begin{array}{c}\text { Hazard } \\
\text { ratio }\end{array} \\
0.987\end{array}$} & \multirow{2}{*}{$\frac{\mathbf{9 5 \%} \mathbf{C I}}{0.499-1.954}$} & \multirow{2}{*}{$\frac{\boldsymbol{P} \text {-value }}{0.971}$} & \multirow[t]{2}{*}{$\begin{array}{l}\text { Hazard } \\
\text { ratio }\end{array}$} & \multirow[t]{2}{*}{$95 \%$ CI } & \multirow[t]{2}{*}{$P$-value } \\
\hline Age, years & $<60$ vs. $\geq 60$ & & & & & & \\
\hline Gender & Female vs. Male & 0.685 & $0.283-1.660$ & 0.403 & & & \\
\hline KPS & 90 vs. 80 or 70 & 0.790 & $0.393-1.588$ & 0.508 & & & \\
\hline Smoke & Never vs. Ever & 1.199 & $0.601-2.391$ & 0.607 & & & \\
\hline COPD & No vs. Yes & 0.517 & $0.213-1.253$ & 0.144 & & & \\
\hline Histology & NSCLC vs. SCLC & 1.839 & $1.905-3.739$ & 0.092 & & & \\
\hline TNM stage & I-II vs. III & 0.689 & $0.345-1.374$ & 0.290 & & & \\
\hline PORT & Yes vs. No & 0.623 & $0.281-1.381$ & 0.244 & & & \\
\hline Dose & $\geq 60$ Gy vs. $50-60$ Gy & 2.495 & $1.227-5.074$ & 0.012 & 2.548 & $1.248-5.202$ & 0.010 \\
\hline V20 & $\geq 20 \%$ vs. $<20 \%$ & 3.617 & $1.396-9.371$ & 0.008 & 2.865 & $1.033-7.944$ & 0.043 \\
\hline MLD & $\geq 12$ Gy vs. $<12$ Gy & 3.835 & $1.664-8.841$ & 0.002 & 2.728 & $1.116-6.666$ & 0.028 \\
\hline NLR & $<2.2$ vs. $\geq 2.2$ & 0.466 & $0.226-0.962$ & 0.039 & 0.406 & $0.196-0.839$ & 0.015 \\
\hline Neoadjuvant CT & Yes vs. No & 1.269 & $0.524-3.073$ & 0.598 & & & \\
\hline Concurrent $\mathrm{CT}$ & Yes vs. No & 1.084 & $0.546-2.150$ & 0.818 & & & \\
\hline Adjuvant CT & Yes vs. No & 0.911 & $0.457-1.818$ & 0.792 & & & \\
\hline
\end{tabular}

Abbreviations: RILI, radiation induced lung injury; CI, confidence interval; KPS, Karnofsky scores; COPD, chronic obstructive pulmonary disease; NSCLC, non-small cell lung cancer; SCLC, small cell lung cancer; PORT, post-operative radiotherapy; V20, volume at least received 20Gy; MLD, mean lung dose; NLR, neutrophil to lymphocyte ratio; CT, chemotherapy.

of RILI are still needed. In current study, we revealed that pre-treatment NLR level, a representative indicator of systemic inflammation, was associated with grade 3 or higher RILI. However, the exact mechanism underlying this effect remains unclear. The most possible reason is the host inflammatory response participates the initiation and progression of RILI [5-7]. As we known, neutrophils could secrete cytokines and chemokines mediate inflammatory cell recruitment and angiogenesis. In addition, an elevated neutrophil could suppress the cytolytic activity of lymphocytes, natural killer cells, activated $\mathrm{T}$ cells, and adaptive immune response suppression $[18,19]$. On the other side, lymphocytes exert a critical role in cytotoxic cell death and cytokine production that reduce inflammation infiltration [20,21]. Some preclinical studies showed that decreasing the amount of neutrophils or macrophages could reduce the amount of lung fibrosis [7, 22, 23]. And these results were also confirmed in clinical setting for the first time in current study. Transforming growth factor beta 1 (TGF- $\beta 1$ ) is a multifunctional growth factor and exerts a critical role in the development of RILI [24-26]. However, many mechanisms on the interaction between neutrophil and TGF- $\beta 1$ have been found $[27,28]$. The interaction between these two factors may also contribute to the interpretation of the role of NLR in RILI prediction.
As a retrospective study, there are a couple of limitations. Firstly, the bias of patient selection and the diagnosis of RILI which based on the medical records may have an impact on the interpretation of the results. Secondly, although we confirmed the predictive value of NLR in this study, the optimal cut-off value of NLR still need more research to establish. Thirdly, poor pulmonary function was thought to be an important patient-related risk factor for the development of RILI [29], nevertheless, pulmonary function tests parameters, such as forced expiratory volume, had not been evaluated in current study for the missing data of some patients. Lastly, the relationship between NLR and other predictive biological markers, such as TGF- $\beta 1$ and interferons (IFNs), interleukin (IL)-6, IL-1, IL-10, and tumor necrosis factor (TNF)- $\alpha$, was not assessed. The combination of these biological markers with NLR may further improve the prediction of RILI.

In conclusion, we demonstrated that total dose $\geq 60 \mathrm{~Gy}, \mathrm{~V} 20 \geq 20 \%, \mathrm{MLD} \geq 12 \mathrm{~Gy}$, and NLR $\geq 2.2$ were independent predictors for the occurrence of grade 3 or higher RILI of lung cancer patients who received IMRT and chemotherapy. And the combined analysis of these factors could improve the predictive ability of RILI, thereby, affording the opportunity to individualize therapy. However, in order to optimal use these easy accessed 
factors in daily practice, validation in a prospective multicenter study is essential.

\section{MATERIALS AND METHODS}

\section{Patient population}

166 lung cancer patients were enrolled in this study. All of the enrolled patients received sequential or concurrent chemoradiotherapy at our hospital between April 2014 and May 2016. 15 patients with coexistent obstructive pneumonia were excluded from the analysis. This investigation was approved by The General Hospital of Ningxia Medical University institutional review board (2016-200).

\section{Treatment}

All patients underwent planning computed tomography (CT) using a Somatom Sensation Open CT scanner (Simens Medical Systems, Munich, Germany). Axial CT images were obtained with a maximum slice separation of $0.5 \mathrm{~cm}$ from the mandible to the lower edge of the liver in the treatment position. The image sets were transferred to the Pinnacle V8.0 treatment planning system (Philips Medical, Madison, WI, USA) for contouring and planning. Following the RTOG recommendations, the target volume and organs at risk structures were contoured on individual simulation CT slices. All contouring was carried out by one radiation oncologist and verified by a second radiation oncologist.
All the irradiation was given using the IMRT technique with tissue inhomogeneity corrections. The collapsed cone algorithm (CC convolution) was used for the final plan calculation and to determine all dosevolume histogram (DVH) values. The prescription dose for the definitive treatment was 60-64 Gy/30-32 fractions, for the postoperative radiotherapy was $50 \mathrm{~Gy} / 25$ fractions. The criterion for acceptance of the plan was that at least $99 \%$ of the planning target volume (PTV) was covered by $95 \%$ of the prescription dose and the maximum dose was less than or equal to $105 \%$. Normal tissue dose-volume constraints were applied. Limited volumes of spinal cord were allowed to exceed $45 \mathrm{~Gy}$. The mean dose of heart were constrained from receiving more than $35 \mathrm{~Gy}$. The mean dose of esophagus was kept from exceeding $34 \mathrm{~Gy}$. The MLD was kept from exceeding 15 Gy, and V20 was kept less than $20 \%$ or lower if possible. The total normal lung volume was defined as the volume of both lungs minus the gross target volume (GTV).

The most used regimens for concurrent chemoradiotherapy consisted of vinorelbine and cisplatin for NSCLC, etoposide and cisplatin for small cell lung cancer (SCLC).

\section{Follow-up}

As the primary endpoint of this study, grade 3 or higher RILI was classified according to Common Terminology Criteria for Adverse Events version 3.0. Patients were assessed weekly during whole radiotherapy procedure. After radiation, all patients were subjected to

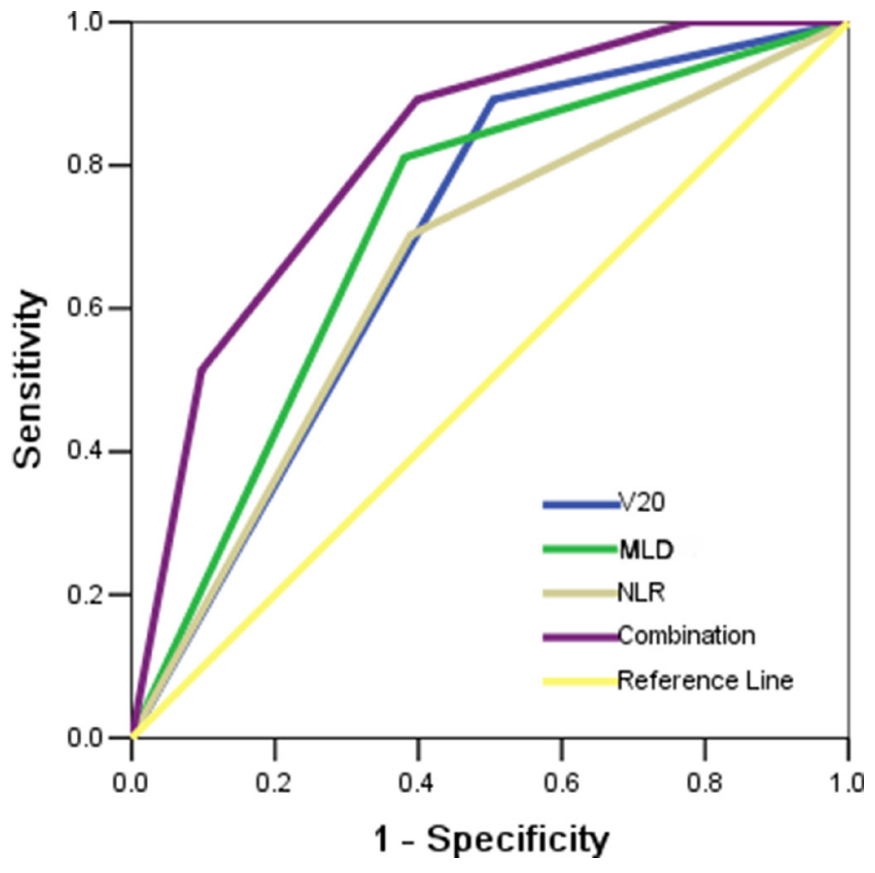

Figure 1: Receiving operator characteristic curve based on the sensitivity and specificity of V20 alone, MLD alone, NLR alone, or all of these three factors combined. 
a follow-up every 2 to 4 weeks for up to 6 months, and then every 3 months for 2 years. History and physical examination, chest CT scan were checked at each followup. RILI was diagnosed according to clinical symptoms, laboratory test results, chest CT scans. The times to endpoints development were calculated from the beginning of radiotherapy; patients not experiencing the endpoint were censored at the last follow-up. The median follow-up time was 15 months (range, 1 to 31 months).

\section{Statistical analysis}

The ROC curve was used to identify the best cut-off points for different variables with which to assess the risk of RILI. The AUC was used to assess the predictive value of each risk factor. Differences in clinical factors and DVH metrics between the higher and lower NLR groups were assessed by a chi-square test or Fisher's exact test where appropriate. Cox proportional hazards analysis was used to calculate the hazard ratio (HR) and confidence interval (CI) to evaluate the influence of clinicopathological and dosimetric variables on the time of RILI development. The analysis performed at the end of follow-up. The meaningful factors which confirmed in univariate analysis were then tested by multivariate analysis. All $P$ values of less than 0.05 were considered statistically significant. Statistical analysis was performed using SPSS 16.0 statistical software package (SPSS Inc, Chicago, IL).

\section{ACKNOWLEDGMENTS AND FUNDING}

This work was supported by a grant from the National Natural Science Foundation of China (81460477) and a research grant from Ningxia Medical University (XM2015061).

\section{CONFLICTS OF INTEREST}

The authors declare no conflicts of interest.

\section{REFERENCES}

1. Khalil AA, Hoffmann L, Moeller DS, Farr KP, Knap MM. New dose constraint reduces radiation-induced fatal pneumonitis in locally advanced non-small cell lung cancer patients treated with intensity-modulated radiotherapy. Acta Oncol. 2015; 54:1343-9.

2. Benveniste MF, Welsh J, Godoy MC, Betancourt SL, Mawlawi OR, Munden RF. New era of radiotherapy: an update in radiation-induced lung disease. Clin Radiol. 2013; 68:e275-90.

3. Vogelius IR, Bentzen SM. A literature-based meta-analysis of clinical risk factors for development of radiation induced pneumonitis. Acta Oncol. 2012; 51:975-83.

4. Ghafoori P, Marks LB, Vujaskovic Z, Kelsey CR. Radiationinduced lung injury. Assessment, management, and prevention. Oncology (Williston Park). 2008; 22:37-47; discussion 52-3.

5. Hill RP, Zaidi A, Mahmood J, Jelveh S. Investigations into the role of inflammation in normal tissue response to irradiation. Radiother Oncol. 2011; 101:73-9.

6. Chen J, Tian X, Mei Z, Wang Y, Yao Y, Zhang S, Li X, Wang H, Zhang J, Xie C. The effect of the TLR9 ligand CpG-oligodeoxynucleotide on the protective immune response to radiation-induced lung fibrosis in mice. Mol Immunol. 2016; 80:33-40.

7. Abernathy LM, Fountain MD, Rothstein SE, David JM, Yunker CK, Rakowski J, Lonardo F, Joiner MC, Hillman GG. Soy Isoflavones Promote Radioprotection of Normal Lung Tissue by Inhibition of Radiation-Induced Activation of Macrophages and Neutrophils. J Thorac Oncol. 2015; 10:1703-12.

8. Li X, Shen J, Lu Z, Chen M, Fang X, Wang G. High neutrophil-to-lymphocyte ratio is associated with increased carotid artery intima-media thickness in type 2 diabetes. $\mathrm{J}$ Diabetes Investig. 2017; 8:101-107.

9. Ozdemir HH, Akil E, Acar A, Tamam Y, Varol S, Cevik MU, Arikanoglu A. Changes in serum albumin levels and neutrophil-lymphocyte ratio in patients with convulsive status epilepticus. Int J Neurosci. 2017; 127:417-420.

10. Berardi R, Rinaldi S, Santoni M, Newsom-Davis T, Tiberi M, Morgese F, Caramanti M, Savini A, Ferrini C, Torniai M, Fiordoliva I, Bower M, Cascinu S. Prognostic models to predict survival in patients with advanced nonsmall cell lung cancer treated with first-line chemo- or targeted therapy. Oncotarget. 2016; 7:26916-24. https://doi. org/10.18632/oncotarget.8309.

11. Lattanzi S, Cagnetti C, Provinciali L, Silvestrini M. Neutrophil-to-Lymphocyte Ratio Predicts the Outcome of Acute Intracerebral Hemorrhage. Stroke. 2016; 47:1654-7.

12. Dong YW, Shi YQ, He LW, Su PZ. Prognostic significance of neutrophil-to-lymphocyte ratio in rectal cancer: a metaanalysis. Onco Targets Ther. 2016; 9:3127-34.

13. Yuan X, Liao Z, Liu Z, Wang LE, Tucker SL, Mao L, Wang XS, Martel M, Komaki R, Cox JD, Milas L, Wei Q. Single nucleotide polymorphism at rs1982073:T869C of the TGFbeta 1 gene is associated with the risk of radiation pneumonitis in patients with non-small-cell lung cancer treated with definitive radiotherapy. J Clin Oncol. 2009; 27:3370-8.

14. Yorke ED, Jackson A, Rosenzweig KE, Braban L, Leibel SA, Ling CC. Correlation of dosimetric factors and radiation pneumonitis for non-small-cell lung cancer patients in a recently completed dose escalation study. Int $\mathrm{J}$ Radiat Oncol Biol Phys. 2005; 63:672-82.

15. Kong FM, Hayman JA, Griffith KA, Kalemkerian GP, Arenberg D, Lyons S, Turrisi A, Lichter A, Fraass B, Eisbruch A, Lawrence TS, Ten Haken RK. Final toxicity results of a radiation-dose escalation study in patients with non-small-cell lung cancer (NSCLC): predictors for radiation pneumonitis and fibrosis. Int J Radiat Oncol Biol Phys. 2006; 65:1075-86. 
16. Graham MV, Purdy JA, Emami B, Harms W, Bosch W, Lockett MA, Perez CA. Clinical dose-volume histogram analysis for pneumonitis after 3D treatment for non-small cell lung cancer (NSCLC). Int J Radiat Oncol Biol Phys. 1999; 45:323-9.

17. Marks LB, Bentzen SM, Deasy JO, Kong FM, Bradley JD, Vogelius IS, El Naqa I, Hubbs JL, Lebesque JV, Timmerman RD, Martel MK, Jackson A. Radiation dosevolume effects in the lung. Int J Radiat Oncol Biol Phys. 2010; 76:S70-6.

18. Mishalian I, Granot Z, Fridlender ZG. The diversity of circulating neutrophils in cancer. Immunobiology. 2017; 222:82-88.

19. Grommes J, Soehnlein O. Contribution of neutrophils to acute lung injury. Mol Med. 2011; 17:293-307.

20. Smith HA, Kang Y. The metastasis-promoting roles of tumor-associated immune cells. J Mol Med (Berl). 2013; 91:411-29.

21. Johnston CJ, Williams JP, Okunieff P, Finkelstein JN. Radiation-induced pulmonary fibrosis: examination of chemokine and chemokine receptor families. Radiat Res. 2002; 157:256-65.

22. Herter JM, Kraft F, Van Aken H, Meersch M, Zarbock A, Rossaint J. GDF-15 prevents ventilator-induced lung injury by inhibiting the formation of platelet-neutrophil aggregates. Thromb Haemost. 2015; 114:434-7.
23. Fox J, Haston CK. CXC receptor 1 and 2 and neutrophil elastase inhibitors alter radiation-induced lung disease in the mouse. Int J Radiat Oncol Biol Phys. 2013; 85:215-22.

24. Anscher MS, Kong FM, Andrews K, Clough R, Marks LB, Bentel G, Jirtle RL. Plasma transforming growth factor beta1 as a predictor of radiation pneumonitis. Int J Radiat Oncol Biol Phys. 1998; 41:1029-35.

25. Sprung $\mathrm{CN}$, Forrester HB, Siva S, Martin OA. Immunological markers that predict radiation toxicity. Cancer Lett. 2015; 368:191-7.

26. Stenmark MH, Cai XW, Shedden K, Hayman JA, Yuan S, Ritter T, Ten Haken RK, Lawrence TS, Kong FM. Combining physical and biologic parameters to predict radiation-induced lung toxicity in patients with non-smallcell lung cancer treated with definitive radiation therapy. Int J Radiat Oncol Biol Phys. 2012; 84:e217-22.

27. Yang L, Pang Y, Moses HL. TGF-beta and immune cells: an important regulatory axis in the tumor microenvironment and progression. Trends Immunol. 2010; 31:220-7.

28. Mancini ML, Sonis ST. Mechanisms of cellular fibrosis associated with cancer regimen-related toxicities. Front Pharmacol. 2014; 5:51.

29. Kong FM, Wang S. Nondosimetric risk factors for radiationinduced lung toxicity. Semin Radiat Oncol. 2015; 25:100-9. 